\title{
Semi-hyperbolicity and bi-shadowing in nonautonomous difference equations with Lipschitz mappings
}

\author{
Phil Diamond $^{\mathrm{a} 1}$, P.E. Kloeden ${ }^{\mathrm{b}} *$ and V.S. Kozyakin ${ }^{\mathrm{c} 2}$ \\ ${ }^{a}$ Department of Mathemtics, University of Queensland, Brisbane, Australia ${ }^{b}$ Fachbereich Mathematik, \\ Johann Wolfgang Goethe Universität, Frankfurt am Main, Germany; ${ }^{c}$ Institute of Information \\ Transmission Problems, Russian Academy of Sciences, Moscow, Russia
}

(Received 12 July 2007; final version received 12 July 2007)

Dedicated to Gerry Ladas on his 70th birthday

\begin{abstract}
It is shown how known results for autonomous difference equations can be adapted to definitions of semi-hyperbolicity and bi-shadowing generalized to nonautonomous difference equations with Lipschitz continuous mappings. In particular, invertibility and smoothness of the mappings are not required and, for greater applicability, the mappings are allowed to act between possibly different Banach spaces.
\end{abstract}

Keywords: hyperbolicity; Lipschitz mappings; bi-shadowing

AMS Subject Classification: 58F15

\section{Introduction}

The role of hyperbolicity and diffeomorphisms in smooth autonomous discrete systems, their associated shadowing properties and formation of chaotic flows is well understood $[13,14,19]$. However, a significant practical problem with the hyperbolicity condition is the difficulty of numerical verification, notably in computer-assisted proofs. Further problems arise if the system is subject to nonsmooth perturbations, or if invertibility is lost. Recent progress has been made by introducing semi-hyperbolic conditions. These are subject to direct numerical verification, share much the same consequences as hyperbolicity, and yet perhaps be neither invertible nor smooth, and are more robust to perturbation. See Refs. [1-9] and references therein for further details.

The concept of shadowing, where every pseudo-trajectory, for example one generated by a computer simulation, is close to some true trajectory, has been seen as guaranteeing that computed behaviour more or less reflects that of the original system. Of equal practical importance is inverse shadowing, where every true orbit can be approximated by some pseudo-trajectory from a class of such, typically generated by a computational method. This problem has been addressed and where both are present, the term bi-shadowing is used. Explicit expressions for the bi-shadowing parameters can be given in terms of the semi-hyperbolic quantities defining a split [1].

Despite all these results and advances in autonomous discrete systems, the nonautonomous case is far less clarified. Only relatively recently have the concepts of cocycles and skew products delivered a context within which powerful analytic techniques have been developed to study such systems (see Refs. [10,16,17] and references therein).

*Corresponding author. Email: kloeden@math.uni-frankfurt.de 
This paper uses the cocycle formalism to extend notions of semi-hyperbolicity and bi-shadowing to nonautonomous systems. Section 2 briefly recalls cocycles and Section 3 uses cocycles to define semi-hyperbolicity in a nonautonomous context. Section 4 defines bi-shadowing in this formalism and contains the principal result. As in the autonomous case, bi-shadowing parameters are expressed in terms of constants defining a split. Indeed, the time-independent proof of Ref. [1], suitably modified for the different definitions, goes through virtually identically in the nonautonomous case. The final section is devoted to a concrete example of a nonsmooth, noninvertible system, to illustrate the techniques and concepts involved.

\section{Nonautonomous difference equations}

Let $P$ be a nonempty space and let $\theta: P \rightarrow P$ be mapping, so that $\left\{\theta^{n}, n \in \mathbb{Z}^{+}\right\}$is a semi-group under composition. No assumption is made about the domain $P$, nor properties of the mapping $p \mapsto \theta p$, but in applications $P$ is often either a metric space (deterministic systems) or a measure space (random systems), with $p \mapsto \theta p$ being continuous in the former and measurable in the latter. In addition, let $\mathfrak{X}=\left\{\left(X_{p},\|\cdot\|_{p}\right), p \in P,\right\}$ be a family of Banach spaces (possibly subspaces of a common space) and let $\mathfrak{f}=\left\{(f(p, \cdot), p \in P\right.$,$\} be a family of mappings f(p, \cdot): X_{p} \rightarrow X_{\theta p}$, $p \in P$, which are at least continuous in $x$.

Consider the nonautonomous difference equation

$$
x_{n+1}=f\left(\theta^{n} p, x_{n}\right),
$$

on $\mathfrak{X}$ with $x_{n} \in X_{\theta^{n} p}$ for each $n \in \mathbb{Z}^{+}$. This generates a discrete-time nonautonomous semidynamical on $\mathfrak{X}$ with the autonomous semi-dynamical system on $P$ generated by $\theta$ as its driving system and the associated cocycle mapping on $\mathfrak{X}$ defined by

$$
\phi(0, p, x):=\{x\} \quad \text { and } \quad \phi(n, p, x):=f\left(\theta^{n} p, \cdot\right) \circ \cdots \circ f(p, x) \quad \text { for all } n \geq 1,
$$

where $\phi(n, p, \cdot): X_{p} \rightarrow X_{\theta ' p}$, see Refs. [14,15].

A simple example is a sequence of mappings $f_{n}$ on $\mathbb{R}^{d}$ with $P=\mathbb{Z}, f(n, \cdot)=f_{n}(\cdot)$, and $\theta$ the left shift on $\mathbb{Z}^{+}$. A nontrivial example arises when the sequence of mappings $f_{n}$ on $\mathbb{R}^{d}$ is chosen periodically or, perhaps less regularly, from a finite family of mappings $\left\{g_{1}, \ldots, g_{r}\right\}$, i.e. with $f_{n}=g_{i_{n}}$ where the $i_{n} \in\{1, \ldots, r\}$. The nonautonomous difference equation

$$
x_{n+1}=g_{i_{n}}\left(x_{n}\right),
$$

on $\mathbb{R}^{d}$ can be rewritten in the form (1) with the set $P=\{1, \ldots, r\}^{\mathbb{Z}^{+}}$of infinite sequences $P=\left\{i_{n}, n \in \mathbb{Z}^{+}\right\}$with $i_{n} \in\{1, \ldots, r\}$ and the left shift operator $\theta$ defined on $P$ by $\theta\left(i_{n}\right)=i_{n+1}$. The space $P$ here may be given the structure of a compact metric space with metric

$$
d\left(p, p^{\prime}\right)=\sum_{n=0}^{\infty}(r+1)^{-|n|}\left|i_{n}-i_{n}^{\prime}\right|
$$

An important application is a variable time-step method for an autonomous differential equation $\dot{x}=f(x)$, such as the Euler method

$$
x_{n+1}=x_{n}+h_{n} f\left(x_{n}\right),
$$

where the variable time steps $h_{n}>0$, in practice, come from a finite set, so $g_{i_{n}}(x)=x+h_{n} f(x)$ in (2) and the sequence space metric is as in (3). More generally, a variable parameter $q \in Q$ 
may be involved in a difference equation $x_{n+1}=f\left(x_{n}, q_{n}\right)$, where $q_{n} \in Q$. For example, let

$$
f(x, q)=\frac{|x|+q^{2}}{1+q}, \quad x \in \mathbb{R}, Q=[0.5,1] .
$$

Here, the sequence space is $P=[0.5,1]^{\mathbb{Z}^{+}}$of infinite sequences $p=\left\{q_{n}, n \in \mathbb{Z}^{+}\right\}, q_{n} \in Q$, which is a compact metric space with the metric

$$
d\left(p, p^{\prime}\right)=\sum_{n=0}^{\infty} 2^{-|n|}\left|q_{n}-q_{n}^{\prime}\right|
$$

A simple example indicating the usefulness of different spaces $X_{p}$ is given in the next section.

\section{Semi-hyperbolicity}

As in the autonomous case, a four-tuple of real numbers called a split measures the expansion, contraction and other rates in the definition of a nonautonomous semi-hyperbolic system (1).

Definition 1. A four-tuple $\mathbf{s}=\left(\lambda_{s}, \lambda_{u}, \mu_{s}, \mu_{u}\right)$ of non-negative real numbers is called a split if

$$
\lambda_{s}<1<\lambda_{u}, \quad\left(1-\lambda_{s}\right)\left(\lambda_{u}-1\right)>\mu_{s} \mu_{u} .
$$

The split $\boldsymbol{s}$ is called positive if all the values $\lambda_{s}, \lambda_{u}, \mu_{s}$ and $\mu_{u}$ are positive.

Note that a four-tuple $\left(\lambda_{s}, \lambda_{u}, \mu_{s}, \mu_{u}\right)$ is a split if and only if the eigenvalues $\Delta_{1}$ and $\Delta_{2}$ of every matrix

$$
\delta=\left[\begin{array}{ll}
\delta_{11} & \delta_{12} \\
\delta_{21} & \delta_{22}
\end{array}\right],
$$

with components satisfying $\left|\delta_{11}\right| \leq \lambda_{s},\left|\delta_{12}\right| \leq \mu_{s},\left|\delta_{21}\right| \leq \mu_{u},\left|\delta_{22}\right| \geq \lambda_{u}$ are real and satisfy $\left|\Delta_{1}\right|<1<\left|\Delta_{2}\right|$.

Clearly, for any given $\lambda_{s}$ and $\lambda_{u}$ satisfying $\lambda_{s}<1<\lambda_{u}$, a four-tuple of non-negative numbers $\mathbf{s}=\left(\lambda_{s}, \lambda_{u}, \mu_{s}, \mu_{u}\right)$ will be a split if the product $\mu_{s} \mu_{u}$ is small enough. The following lemma from Ref. [4], Lemma 3.1.3, illustrates the degree to which the elements of a split can be perturbed while leaving the resulting four-tuple of perturbed numbers a split.

Lemma 1. Let $\mathbf{s}=\left(\lambda_{s}, \lambda_{u}, \mu_{s}, \mu_{u}\right)$ be a split. Define where

$$
\nu(\mathbf{s}):=\frac{\left(1-\lambda_{s}\right)\left(\lambda_{u}-1\right)-\mu_{s} \mu_{u}}{\lambda_{u}-\lambda_{s}+\mu_{s}+\mu_{u}}
$$

and note that

$$
\nu(\mathbf{s}) \leq \frac{\left(1-\lambda_{s}\right)\left(\lambda_{u}-1\right)}{\lambda_{u}-\lambda_{s}} \leq \min \left\{1-\lambda_{s}, \lambda_{u}-1\right\},
$$


so $\nu(s)<1$. Then, for

$$
\tilde{\lambda_{s}}=\lambda_{s}+d_{1}, \quad \tilde{\lambda_{u}}=\lambda_{u}-d_{2}, \quad \tilde{\mu_{s}}=\mu_{s}+d_{3}, \quad \tilde{\mu_{u}}=\mu_{u}+d_{4},
$$

and real $d_{i}$ satisfying $0 \leq d_{i},<\nu(\mathbf{s})$ for $i=1, \ldots, 4$, the four-tuple $\tilde{\mathbf{s}}=\left(\tilde{\lambda_{s}}, \tilde{\lambda_{u}}, \tilde{\mu_{s}}, \tilde{\mu_{u}}\right)$ is also a split.

Let $\mathfrak{f}=\left\{(f(p, \cdot), p \in P\}\right.$ a family of mappings with $f(p, \cdot): X_{p} \rightarrow X_{\theta p}$ for $p \in P$ and let $\Re=\left\{K_{p}, p \in P\right\}$ be a family of closed and bounded subsets with $K_{p} \subset X_{p}$ and $f\left(p, K_{p}\right) \cap$ $K_{\theta p} \neq \emptyset$ for each $p \in P$.

Definition 2. A splitting $X_{p}=E_{x}^{s}(p) \oplus E_{x}^{u}(p)$ of a family of Banach spaces $\mathfrak{X}=\left\{\left(X_{p},\|\cdot\|_{p}\right)\right.$, $p \in P$,$\} with projectors P_{x}^{s}(p): X_{p} \mapsto E_{x}^{s}(p)$ and $P_{x}^{u}(p): X_{p} \mapsto E_{x}^{u}(p)$ for each $x \in X_{p}$ such that

$$
\begin{aligned}
& P_{x}^{s}(p) X_{p}=E_{x}^{s}(p), \quad P_{x}^{s}(p) E_{x}^{u}(p)=0, \\
& P_{x}^{u}(p) X_{p}=E_{x}^{u}(p), \quad P_{x}^{u}(p) E_{x}^{s}(p)=0,
\end{aligned}
$$

is said to be uniform on the family $\Omega=\left\{K_{p}, p \in P\right\}$ of closed and bounded subsets with respect to the family of mappings $\mathfrak{f}=\left\{(f(p, \cdot), p \in P\right.$,$\} on \mathfrak{X}$ with $f\left(p, K_{p}\right) \cap K_{\theta p} \neq \emptyset$ for each $p \in P$ if there exist positive real numbers $\delta$, $h$ such that

SH0: $\operatorname{dim} E_{y}^{u}(\theta p)=\operatorname{dim} E_{x}^{u}(p)$ for all $x \in K_{p}$ and $y \in K_{\theta p}$ such that $\left.\| f(p, x)-y\right) \|_{\theta p} \leq \delta$ for every $p \in P$;

SH1: $\sup _{p \in P} \sup _{x \in K_{p}}\left\{\left\|P_{x}^{u}(p)\right\|_{p},\left\|P_{x}^{s}(p)\right\|_{p}\right\} \leq h$ for every $p \in P$.

The continuity in $x$ of the splitting subspaces $E_{x}^{s}(p), E_{x}^{u}(p)$ or of the projectors $P_{x}^{s}(p), P_{x}^{u}(p)$ is not assumed in the above definition, nor is the invariance of the subsets $K_{p} \in \mathfrak{R}=\left\{K_{p}\right.$, $p \in P\}$ with respect to the corresponding mappings $f(p, \cdot) \in \mathfrak{K}=\{(f(p, \cdot), p \in P$,$\} . That is,$ neither $K_{\theta_{p}}=f\left(p, K_{p}\right)$ nor $K_{\theta_{p}} \subseteq f\left(p, K_{p}\right)$ has to hold for any $p \in P$. This allows considerably more flexibility in applications, see for example Refs. [6,8].

Definition 3. A family of mappingss $\mathfrak{f}=\{f(p, \cdot), p \in P\}$ on a family of Banach spaces $\mathfrak{X}=\left\{X_{p}, p \in P\right\}$ is said to be uniformly $\mathbf{s}$-semi-hyperbolic with a split $\mathbf{s}=\left(\lambda_{s}, \lambda_{u}, \mu_{s}, \mu_{u}\right)$ on a family $\Omega=\left\{K_{p}, p \in P\right\}$ of nonempty closed and bounded subsets of $\mathfrak{X}$ such that $f\left(p, K_{p}\right) \cap$ $K_{\theta_{p}} \neq \emptyset$ for all $p \in P$, if there exists a uniform splitting $X_{p}=E_{x}^{s}(p) \oplus E_{x}^{u}(p)$ of $\mathfrak{X}$ with projectors $P_{x}^{s}(p)$ and $P_{x}^{u}(p)$ for each $x \in K_{p}$ and $p \in P$ (i.e. satisfying conditions SH0 and SH1) such that

SH2 for each $p \in P$ the inclusion $x+u+v \in X_{p}$ and the inequalities

$$
\begin{aligned}
& \left\|P_{y}^{s}(\theta p)[f(p, x+u+v)-f(p, x+\tilde{u}+v)]\right\|_{\theta p} \leq \lambda_{s}\|u-\tilde{u}\|_{p}, \\
& \left\|P_{y}^{s}(\theta p)[f(p, x+u+v)-f(p, x+u+\tilde{v})]\right\|_{\theta p} \leq \mu_{s}\|v-\tilde{v}\|_{p}, \\
& \left\|P_{y}^{u}(\theta p)[f(p, x+u+v)-f(p, x+\tilde{u}+v)]\right\|_{\theta p} \leq \mu_{u}\|u-\tilde{u}\|_{p}, \\
& \left\|P_{y}^{u}(\theta p)[f(p, x+u+v)-f(p, x+u+\tilde{v})]\right\|_{\theta p} \geq \lambda_{u}\|v-\tilde{v}\|_{p} .
\end{aligned}
$$

hold for all $x \in K_{p}$ and $y \in K_{\theta p}$ with $\left.\| f(p, x)-y\right) \|_{\theta p} \leq \delta$ and all $u, \tilde{u} \in E_{x}^{s}(p)$ and $v, \tilde{v} \in$ $E_{x}^{u}(p)$ with $\|u\|_{p},\|\tilde{u}\|_{p},\|v\|_{p},\|\tilde{v}\|_{p} \leq \delta$. 
A simple example can be constructed in terms of a sequence of linear mappings $f_{n}(x)=A_{n} x$ in $\mathbb{R}^{d}$ with hyperbolic matrices $A_{n}$ for which the unstable linear spaces all have the same dimension. This can be put into formalism with $P=\mathbb{Z}$ and $\theta$ the left shift on $\mathbb{Z}^{+}$with all $X_{n}=\mathbb{R}^{d}$. If the unstable subspaces are each mapped onto their successor, a split with $\mu_{s}=\mu_{u}=0$ can be used.

To see why it may be convenient to distinguish the elements $X_{p}$ of $\mathfrak{X}$, consider a real symmetric $2 \times 2$ hyperbolic matrix $A_{0}$ and a $2 \times 2$ rotation matrix $R$ by $\pi / 2$ and consider a nonautonomous linear difference equation with matrices $A_{n}=R^{n} A_{0}$ for $n \in \mathbb{Z}^{+}$, so $P=\mathbb{Z}^{+}$. Suppose that the space $\mathbb{R}^{2}$ has a fixed coordinate system and define $X_{n}:=R^{n} \mathbb{R}^{2}$, so $X_{n+1}=R X_{n}$. Note that the stable and unstable manifolds would be mapped one into the other if the spaces were not also rotated. Here a split with $\mu_{s}=\mu_{u}=0$ is also appropriate. This example is, of course, trivial - one could avoid the rotations altogether and consider simply the autonomous linear difference equation with matrix $A_{0}$. A less trivial example will be given in Section 5. Discrete time random dynamical systems also provide another important class of such examples, see Imkeller and Kloeden [12].

\section{Shadowing}

The relationship between the behaviour of a given dynamical system and its perturbed counterparts is also important for nonsmooth and noninvertible systems. For example, a computer simulation of a specific dynamical system is really only an approximation of the given system because of the finiteness of computer arithmetic and subsequent round off error. In particular, computed trajectories are only pseudo-trajectories of the original system. This relationship is, however, difficult to express in terms of conjugacy, as is possible for diffeomorphic systems. A convenient and fruitful substitute for a conjugacy relationship in such a situation is provided by the notion of bi-shadowing, in which trajectories and pseudotrajectories are compared rather than the mappings themselves. The concept of bi-shadowing was introduced in Refs. [5,6], see also the forthcoming monograph [4]. It combines the usual concept of direct shadowing with a newer concept of inverse shadowing.

A typical statement of such problems can be formulated in the following general, if somewhat vague form. Given a dynamical system, assumed autonomous for simplicity, $x_{n+1}=f\left(x_{n}\right)$ generated by a mapping $f$ on a metric or topological space $X$, suppose that the function $f$ can be perturbed at any instant $n$ so that another system $y_{n+1}=g\left(n, y_{n}\right)$ is in fact observed, where $g$ is close to $f$ in some sense and can possibly also vary with $n$. The following Direct Shadowing Problem can be posed: for every trajectory of every small perturbation $g$ of a given mapping $f$ does there exists a close trajectory of $f$ ? Allowing nonautonomous perturbations $g$ of the mapping $f$ provides considerably more generality and applicability. The Direct Shadowing Problem is usually formulated in terms of relationships between true trajectories and the pseudo-trajectories of the original system $f$.

The Inverse Shadowing Problem is also of interest: can every trajectory of a mapping $f$ be approximated, or shadowed by a trajectory of any perturbation $\mathrm{g}$ of $f$ from a predefined class $\mathcal{F}$ ? Here the class of perturbations $\mathcal{F}$ is usually restricted to a rather small class of mappings, in some sense, to obtain strong and significant results. For example, the class $\mathcal{F}$ may consist of all continuous autonomous mappings $g$ or, of all mappings, possibly discontinuous, arising in some approximation problem or numerical method.

Bi-shadowing conceptualizes the robustness to perturbations of observed dynamical behaviour of a dynamical system, such as those appearing in computer simulations. It can also be interpreted as a form of dynamical structural stability when restricted to specific classes of mappings, such as continuous mappings. Moreover, it implies both the direct shadowing and the inverse shadowing properties. 


\subsection{Definitions}

As above, let $\Re=\left\{K_{p}, p \in P\right\}$ be a family of nonempty closed and bounded subsets with $K_{p} \subset X_{p}$ for each $p \in P$ such that $f\left(p, K_{p}\right) \cap K_{\theta p} \neq \emptyset, p \in P$, for a family of mappings $\mathfrak{f}=\{f(p, \cdot), p \in P$,$\} with f(p, \cdot): X_{p} \rightarrow X_{\theta p}, p \in P$.

DeFINITION 4. A trajectory of a family of mappings $\mathfrak{f}$ on $\mathfrak{X}$ is a sequence $\mathbf{x}=\left\{x_{n}\right\}$ with $x_{n} \in X_{\theta^{n}} p$ satisfying

$$
x_{n+1}=f\left(\theta^{n} p, x_{n}\right) .
$$

A $\gamma$-pseudo-trajectory of a family of mappings $\mathfrak{\mathfrak { f }}$ on $\mathfrak{X}$ is a sequence $\boldsymbol{y}=\left\{y_{n}\right\}$ with $y_{n} \in X_{\theta^{\prime \prime}}$ satisfying

$$
\left\|y_{n+1}-f\left(\theta^{n} p, y_{n}\right)\right\|_{\theta^{n+1}} \leq \gamma, \quad \gamma>0,
$$

for $n=-n_{-}, \ldots, 0, \ldots, n_{+}$where $n_{ \pm} \leq \infty$. The qualifier finite may be used when $n_{ \pm}<\infty$ and infinite otherwise.

Given a finite or infinite interval $\llbracket$ of integers $\mathbb{Z}$, let $\operatorname{Tr}_{\mathbb{1}}(\mathfrak{f}, \Omega, \gamma)$ denotes the totality of corresponding $\gamma$-pseudo-trajectories of $\mathfrak{f}$ in $\Omega$. Since a true trajectory can be regarded as a $\gamma$-pseudo-trajectory for any $\gamma \geq 0$, in particular with $\gamma=0$, denotes the corresponding set of

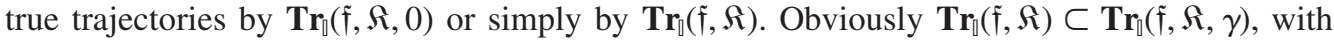
strict inclusion since there are $\gamma$-pseudo-trajectories which are not trajectories.

Let $\mathfrak{g}=\{g(p, \cdot), p \in P$,$\} with g(p, \cdot): X_{p} \rightarrow X_{\theta p}$ for each $p \in P$. The semi-norm

$$
\|\mathfrak{g}-\mathfrak{f}\|_{\infty}=\sup _{p \in P} \sup _{x \in X_{p}}\|g(p, x)-f(p, x)\|_{\theta p}
$$

gives a notion of distance between families $\mathfrak{g}$ and $\mathfrak{f}$ on $\mathfrak{X}$.

DeFINITION 5. A nonautonomous semi-dynamical system generated by a family of Lipschitz mappings $\mathfrak{\uparrow}=\{f(p, \cdot), p \in P$,$\} with f(p, \cdot): X_{p} \rightarrow X_{\theta p}$, such that $f(p, \cdot): X_{p} \rightarrow X_{\theta p}$ for a family $\mathfrak{R}=\left\{K_{p}, p \in P\right\}$ of nonempty closed and bounded subsets with $K_{p} \subset X_{p}$ and $f\left(p, K_{p}\right) \cap K_{\theta p} \neq$ $\emptyset, p \in P$, is said to be bi-shadowing with respect to completely continuous perturbations on $\mathfrak{R}$ with positive parameters $\alpha$ and $\beta$, if for any given finite pseudo-trajectory $\mathbf{x}=\left\{x_{n}\right\} \in$ $\operatorname{Tr}_{0}(\mathfrak{f}, \mathcal{R}, \gamma)$ with $0 \leq \gamma \leq \beta$ and any family of completely continuous mappings $\mathfrak{g}=\{g(p, \cdot), p \in P$,$\} with g(p, \cdot): X_{p} \rightarrow X_{\theta p}, p \in P$, satisfying

$$
\|\mathfrak{g}-\mathfrak{f}\|_{\infty} \leq \beta-\gamma,
$$

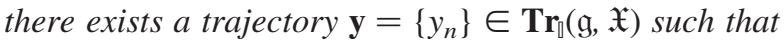

$$
\left\|x_{n}-y_{n}\right\|_{p} \leq \alpha\left(\gamma+\|\mathfrak{g}-\mathfrak{f}\|_{\infty}\right)=\alpha \beta,
$$

for all $n$ for which $\boldsymbol{y}$ is defined.

If the spaces $X_{p}$ are finite-dimensional of like dimension, then the requirement that the sets $K_{p}$ are closed and bounded is equivalent to compactness. The condition that the perturbed mappings $\mathfrak{g}$ are completely continuous, appearing in the definition of bi-shadowing is then equivalent to continuity. 


\subsection{Semi-hyperbolicity implies bi-shadowing}

The main result of the paper is that semi-hyperbolicity is sufficient to ensure bi-shadowing of a nonautonomous semi-dynamical system generated by a family $\mathfrak{f}$ of Lipschitz mapping with respect to a class of perturbed systems generated by families $\mathfrak{g}$ of continuous mappings. As with the corresponding result for autonomous systems, which it generalizes, it also provides explicit values of the shadowing parameters.

THEOREM 1. Let $\mathfrak{\Re}=\left\{K_{p}, p \in P\right\}$ be a family of closed and bounded subsets of $\mathfrak{X}=\left\{X_{p}, p \in P\right\}$ for a family $\mathfrak{f}=\{f(p, \cdot), p \in P\}$ of Lipschitz mappings with $f(p, \cdot): X_{p} \rightarrow X_{\theta p}, p \in P$, which is semi-hyperbolic on $\mathfrak{\Omega}$ with a uniform split $\mathbf{s}$ and constants $h, \delta$. Then, the nonautonomous semidynamical system generated by $\mathfrak{f}$ is bi-shadowing on $\mathfrak{R}$ with respect to any family $\mathfrak{g}=\{g(p, \cdot), p \in P\}$ of completely continuous mappings $g(p, \cdot): X_{p} \rightarrow X_{\theta p}, p \in P$, with parameters $\alpha=\alpha(\mathbf{s}, h)$ and $\beta=\beta(\mathbf{s}, h, \delta)$ defined by

$$
\alpha(\mathbf{s}, h):=h \frac{\lambda_{u}-\lambda_{s}+\mu_{s}+\mu_{u}}{\left(1-\lambda_{s}\right)\left(\lambda_{u}-1\right)-\mu_{s} \mu_{u}} ;
$$

and

$$
\beta(\mathbf{s}, h, \delta):=\delta h^{-1} \frac{\left(1-\lambda_{s}\right)\left(\lambda_{u}-1\right)-\mu_{s} \mu_{u}}{\max \left\{\lambda_{u}-1+\mu_{s}, 1-\lambda_{s}+\mu_{u}\right\}} .
$$

Moreover, if each set $K_{p}$ is compact, then the bi-shadowing holds with respect to trajectories on infinite intervals.

The proof follows very closely those given in Refs. [6,7] for the autonomous case, noting only that all estimates hold uniformly in $p \in P$, and will not be repeated here. See in particular the proofs of Theorems 6.3.3 and 6.3.9 in the forthcoming monograph [4].

A particular strength of the result is that it does not require smooth perturbations and is thus applicable to, for example, perturbations due to hysteresis effects [8]. It also provides explicit error bounds. An important application is when a variable time-step numerical method such as the Euler method is applied to an autonomous differential equation. Due to the discreteness of computer arithmetic, which causes round-off error, an approximation of the Euler mapping is in fact computed $[3,6]$. The bi-shadowing result ensures that the computed dynamics reflects that of the Euler method.

\section{Twisted horseshoe mapping}

The twisted horseshoe mapping $f=\left(f_{1}, f_{2}\right)$ on $I^{2}=[0,1] \times[0,1]$ defined by

$$
f_{1}(x, y)=\left\{\begin{array}{ll}
2 x & \text { for } 0 \leq x \leq \frac{1}{2}, \\
2-2 x & \text { for } \frac{1}{2}<x \leq 1,
\end{array} \quad f_{2}(x, y)=\frac{x}{2}+\frac{y}{10}+\frac{1}{4}\right.
$$

has been investigated by Guckenheimer et al. [11]. It has a fixed point $(\bar{x}, \bar{y})=(2 / 3,35 / 54)$, which is a saddle point with eigenvalues -2 and $1 / 10$. All the conditions of a theorem in Ref. [15] which generalizes Marotto's snap-back repeller result to saddle points are thus satisfied, so the twisted horseshoe mapping behaves chaotically in a slight generalization of the sense of $\mathrm{Li}$ and Yorke (see [18]). In fact, the twisted-horseshoe mapping also satisfies the Sharkovsky 
cycle coexistence ordering, due to a generalization in Ref. [14] of Sharkovsky's theorem to triangular shaped mappings like the twisted horseshoe mapping.

The autonomous difference equation generated by the twisted horseshoe mapping can be interpreted as a nonautonomous difference equation

$$
x_{n+1}=\frac{x_{n}}{10}+\frac{p_{n}}{2}+\frac{1}{4},
$$

driven by the semi-dynamical system on $[0,1]$

$$
p_{n+1}= \begin{cases}2 p_{n} & \text { for } 0 \leq p_{n} \leq \frac{1}{2} \\ 2-2 p_{n} & \text { for } \frac{1}{2}<p_{n} \leq 1 .\end{cases}
$$

Note that any trajectory of the driving system can be extended backwards indefinitely, though typically not uniquely. Let $\mathbf{p}=\left\{p_{n}: n \in \mathbb{Z}\right\}$ be such an entire trajectory and hold it fixed. Then the explicit solution of (13) given by

$$
x_{n}=10^{-n+n_{0}} x_{n_{0}}+\frac{1}{2} \sum_{j=n_{0}}^{n-1} 10^{j+1-n} p_{j}+\frac{1}{4} \sum_{j=n_{0}}^{n-1} 10^{j+1-n} .
$$

Let $n$ fixed and let the $x_{n_{0}}$ take values in a fixed bounded subset of $\mathbb{R}$. Then, taking the pullback limit as $n_{0} \rightarrow-\infty$ (see $\left.[16,17]\right)$, gives

$$
\bar{x}_{n}=\frac{1}{2} \sum_{j=-\infty}^{n-1} 10^{j+1-n} p_{j}+\frac{1}{4} \sum_{j=-\infty}^{n-1} 10^{j+1-n}
$$

that is,

$$
\bar{x}_{n}=\frac{1}{2} \sum_{j=-\infty}^{n-1} 10^{j+1-n} p_{j}+\frac{10}{36} .
$$

In particular, for two different initial values $x_{n_{0}}$ and $x_{n_{0}}^{\prime}$, with the same driving sequence $\mathbf{p}$, it is easily shown that

$$
\left|x_{n}-x_{n}^{\prime}\right|=\left|x_{n_{0}}-x_{n_{0}}^{\prime}\right| 10^{-n+n_{0}} \rightarrow 0 \quad \text { as } n \rightarrow \infty,
$$

and hence, with $x_{n}^{\prime} \equiv \bar{x}_{n}$,

$$
\left|x_{n}-\bar{x}_{n}\right| \rightarrow 0 \quad \text { as } n \rightarrow \infty \text {. }
$$

Thus all solutions of (13) with different starting points converge to the same limit if they correspond to the same driving sequence.

Define $\bar{x}(\mathbf{p}):=\bar{x}_{0}$ and let $\theta$ be the shift operator on the space of sequences $P=[0,1]^{\mathbb{Z}^{+}}$. Then $\bar{x}_{n}=\bar{x}\left(\theta_{n} \mathbf{p}\right)$ and the singleton subsets $A_{\mathbf{p}}:=\{\bar{x}(\mathbf{p})\}$ for the pullback attractor (which is also forwards attracting) of the nonautonomous dynamical system generated by the twisted horseshoe system. Let $X_{\mathbf{p}}$ be the space $\mathbb{R}$ with the origin shifted to $\bar{x}(\mathbf{p})$. With the change 
of coordinates $z_{n}:=x_{n}-\bar{x}(\mathbf{p})$ the mapping (13) simplifies to

$$
z_{n+1}=\frac{1}{10} z_{n}
$$

with $z_{n} \in X_{\theta^{n} \mathbf{p}}$ and $z_{n+1} \in X_{\theta^{n+1}} \mathbf{p}$. Semi-hyperbolicity on $K_{\mathbf{p}} \equiv X_{\mathbf{p}}$ for the split $\mathbf{s}=\left(10^{-1}, 0,0,0\right)$ and trivial unstable subspaces follows immediately. The bi-shadowing Theorem 1 is applicable to this NDS with continuous perturbations of the linear mapping (13).

\section{Notes}

1. Email: pmd@maths.uq.edu.au

2. Email: kozyakin@iitp.ru

\section{References}

[1] A. Al-Nayef, P. Diamond, P.E. Kloeden, V. Kozyakin, and A. Pokrovskii, Bi-shadowing and delay equations, Dyn. Stab. Syst. 10 (1996), pp. 121-129.

[2] A. Al-Nayef, P.E. Kloeden, and A. Pokrovskii, Expansivity of nonsmooth functional differential equations, J. Math. Anal. Appl. 208 (1997), pp. 453-461.

[3] P. Diamond and P.E. Kloeden, Spatial discretization of mappings, J. Comput. Math. Appl. 26 (1993), pp. 85-94.

[4] P. Diamond, P.E. Kloeden, V. Kozyakin, and A. Pokrovskii, Semi-Hyperbolic Mappings and Bi-Shadowing, manuscript to be submitted.

[5] — Expansivity of semi-hyperbolic Lipschitz mappings, Bull. Austral. Math. Soc. 51 (1995), pp. 301-308.

[6] - Computer robustness of semi-hyperbolic mappings, Random Comput. Dyn. 3 (1995), pp. $57-70$

[7] —-, Semi-hyperbolic mappings, J. Nonlinear Sci. 5 (1995), pp. 419-431.

[8] P. Diamond, P.E. Kloeden, V. Kozyakin, M.A. Krasnosel'skii, and A. Pokrovskii, Robustness of dynamical systems to a class of nonsmooth perturbations, Nonlinear Anal. TMA 26 (1996), pp. 351-361.

[9] P. Diamond, P.E. Kloeden, M.A. Krasnosel'skii, and A. Pokrovskii, Chaotic dynamics in nonsmooth perturbations of bishadowing systems, Arab J. Math. Sci. 6 (2000), pp. 41-74.

[10] P. Glendinning, Global attractors of pinched skew products, Dyn. Syst. 17(3) (2002), pp. 287-294.

[11] J. Guckenheimer, G. Oster, and A. Ipaktachi, The dynamics of density dependent population models, J. Math. Biol. 4 (1977), pp. 101-147.

[12] P. Imkeller and P.E. Kloeden, On the computation of invariant measures in random dynamical systems, Stoch. Dyn. 3 (2003), pp. 247-265.

[13] A. Kotak and B. Hasselblatt, Modern Theory of Dynamical Systems, Cambridge University Press, Cambridge, 1995.

[14] P.E. Kloeden, On Sharkovsky's cycle coexistence ordering, Bull. Austral. Math. Soc. 20 (1979), pp. $171-178$.

[15] - Chaotic difference equations in $\Re^{n}$, J. Austral. Math. Soc. 31A (1981), pp. 217-225.

[16] — Pullback attractors of nonautonomous semidynamical systems, Stoch. Dyn. 3(1) (2003), pp. $101-112$.

[17] $\longrightarrow$, Pullback attractors in nonautonomous difference equations, J. Difference Equ. Appl. 6 (2000), pp. 33-52.

[18] P.E. Kloeden and Z. Li, Beyond the Li-Yorke definition of chaos, J. Difference Equ. Appl. 12 (2006), pp. 247-269.

[19] J. Palis and F. Takens, Hyperbolicity and sensitive chaotic dynamics at homoclinic bifurcations. Cambridge Studies in Advanced Mathematics. Vol. 35. Cambridge: Cambridge University Press. $\mathrm{x}+234 \mathrm{pp}$. 